INTERNATIONAL JOURNAL OF APPLIED AND COMPUTATIONAL MATHEMATICS.

Accepted January $29^{\text {th }}, 2018$

ISSN: 2349-5103 (Print) 2199-5796 (Online)

Publisher: Springer

\title{
NATURAL CONVECTION IN A SQUARE CAVITY WITH UNIFORMLY HEATED AND/OR INSULATED WALLS USING MARKER-AND-CELL METHOD
}

\author{
B MD HIDAYATHULLA KHAN* \\ Department of Mathematics, Jawaharlal Nehru Technological University Ananthapur, Anantapuramu - 515 002 , India. \\ O. ANWAR BÉG \\ Aeronautical and Mechanical Engineering Department, School of Computing, Science and Engineering, Newton Building, \\ University of Salford, M54WT, UK. \\ V RAMACHANDRA PRASAD \\ Department of Mathematics, Madanapalle Institute of Technology \& Science, Madanapalle - 517 325, India. \\ R BHUVANA VIJAYA \\ Department of Mathematics, Jawaharlal Nehru Technological University Ananthapur, Anantapuramu - 515002 , India. \\ *Corresponding author Email: bmdhkh@gmail.com
}

\begin{abstract}
In this study, a numerical investigation has been performed using the computational HarlowWelch MAC (Marker and Cell) finite difference method to analyse the unsteady state twodimensional natural convection in lid-driven square cavity with left wall maintained at constant heat flux and remaining walls are kept thermally insulated. The significant parameters in the present study are Reynolds number $(R e)$, thermal Grashof number $(G r)$ and Prandtl number $(\mathrm{Pr})$ and Peclét number $(\mathrm{Pe}=\mathrm{PrRe})$. The structure of thermal convection patterns is analysed via streamline, vorticity, pressure and temperature contour plots. The influence of the thermophysical parameters on these distributions is described in detail. Validation of solutions with earlier studies is included. Mesh independence is also conducted. It is observed that an increase in Prandtl number intensifies the primary circulation whereas it reduces the heat transfer rate. Increasing thermal Grashof number also decreases heat transfer rates. Furthermore the isotherms are significantly compressed towards the left (constant flux) wall with a variation in Grashof number while Peclét number is fixed. The study is relevant to solar collector heat transfer simulations and also crystal growth technologies.
\end{abstract}

KEY WORDS: Enclosure; thermal convection; MAC numerics; Thermal Grashof number; Lid-driven; vorticity; isotherms; Prandtl number; solar collectors.

\section{INTRODUCTION}

The analysis of natural convection in enclosures (cavities) plays an important role in numerous branches of engineering technology and applied sciences. These include fire dynamics [1], 
solar energy collector performance [2], geophysical systems [3], crystal fabrication [4] and bioreactor design [5] and the nature of the enclosure may vary from rectangular to elliptic, circular, triangular, annular or more complex geometries. Natural (free) convection flows are characterized by non-trivial buoyancy forces. The interplay between buoyancy and inertial forces exerts a profound influence on flow and thermal structural development. Among closed cavity geometries, free convection heat transfer in a rectangular cavity is of particular interest in drying technology, electronics device cooling and solar cells. For the case of aspect ratio of unity, the square cavity is retrieved. Both theoretical and experimental studies of transport in rectangular enclosures have been reported in recent years considering a multiplicity of thermophysical and other body force effects. Rudraiah et al. [6] studied the effect of a magnetic field on free convection inside a rectangular enclosure. They found that a circular flow was formed with a relatively weak magnetic field, the convection was suppressed and the rate of convective heat transfer decreased when the magnetic field strength was increased. Garandet et al. [7] analysed the effect of a magnetic field on buoyancy driven convection in a rectangular enclosure. Bhuvaneswari et al. [8] investigated the numerical solution of the mixed convection flow with Soret (thermo-diffusion) effect in a two-sided lid-driven square cavity. They observed that the heat and mass transfer rate decreases with greater Richardson number. Moallemi et al [9] studied the effect of Prandtl number on the mixed convection in a lid-driven cavity with lower wall heated and insulated side walls. They found that the impact of buoyancy is amplified for large values of Prandtl number. Sathiyamoorthy et al. [10] studied the free convective electrically-conducting flow in a square cavity for uniformly heated adjacent walls with magnetic body force, observing that local and average Nusselt number are considerably modified with magnetic field. Kakarantzas et al. [11] used a direct numerical method with a fractional semi-implicit scheme approach to simulate natural hydromgnetic convection in a vertical cylindrical cavity with sinusoidal top wall temperature variation. Saravanan et al. [12] examined numerically the two-dimensional buoyancy-driven convection in a square cavity with isoflux and isothermal boundary conditions. Walker and Homsy [13] investigated buoyancy-driven convection in a porous cavity. Abdul Hakeema et al. [14] analysed the free convection in an air filled square enclosure with thermally active boundaries for different boundary conditions.

Modification in the heat transfer characteristics in cavities via the introduction of isothermal fins has also stimulated some interest. Shi and Khodadadi [15] and Lakhal et al. [16] have discussed the impact of adding isothermal fins to one of the active walls in 
enclosures. They identified flow patterns changed by the hydrodynamic blockage impact depending upon the length of the fin and an extra heating of the fluid that is offered by the fin. In addition the extra heating mechanism nullifies the hydrodynamic blockage impact and contributed to the strengthening of the flow field for high Rayleigh numbers. Conjugate conduction-convection in differentially heated cavities containing a conducting or heat generating strong block is also addressed in other studies [17-19]. These studies have shown that the heat transfer processes in cavities can be balanced by changing the physical and geometrical requirements. Roy et al. [20] studied the mixed convection in lid-driven porous square cavities with various heating conditions on the horizontal walls and elaborated on the influence of Peclét number. Basak et al. [21] used a finite element method to analyse the heat transfer and entropy generation in free convection in tilted square enclosures, observing that low entropy generation occurs with an inclination of $15^{\circ}$ in the convection dominant mode irrespective of Prandtl number. Ismael et al. [22] studied the influence of wall slip on combined free and forced convection in a lid-driven square enclose with a vertical adiabatic wall. Bhattacharya et al. [18] examined the flow structure and temperature patterns during mixed convection in a lid-driven trapezoidal cavity with a cold top moving wall and the bottom wall being heated by isothermally and non-isothermally. They found that a non-isothermal horizontal wall leads to multiple steady states in either the natural convection dominated regime or mixed convection regime. Free convection in a lid-driven square cavity induced by two mutually perpendicular heated walls has also been considered by other researchers [24, 25].

The vast majority of the above studies have ignored unsteadiness effects and been confined to single Prandtl numbers. The aim of the present study is to simulate transient natural convection in a square cavity with the left side wall uniformly heated (or constant heat flux), and the other three walls being thermally insulated. The transformed, dimensionless conservation partial differential equations for momentum and energy are strongly non-linear. A computational Marker and Cell finite difference scheme (MAC) based on the original methodology due to Welch and Harlow [26] is therefore adopted to solve the non-linear boundary value problem to determine the pressure, velocity, vorticity and temperature distributions in the square enclosure. The influence of Prandtl number, Grashof number and Reynolds number is explored. The paper is structured as follows. Section 2 describes the mathematical model. Section 3 presents numerical simulation details. Section 4 elaborates on computational visualizations and interpretation. Section 5 summarizes the main findings. The 
present investigation is relevant to further elucidating the time-dependent thermofluid dynamics in for example square solar collectors.

\section{MATHEMATICAL MODELLING AND SIMULATION}

The regime under investigation is illustrated in Fig. 1. We consider a two-dimensional square cavity with physical dimensions where the left wall is maintained at constant heat flux and the remaining three walls are kept thermally insulated. The top wall is assumed to slide from left to right with constant velocity, $U_{0}$. Thermo-physical properties of the fluid such as thermal conductivity, viscosity, specific heat, permeability and thermal expansion coefficient treated as constant. The Boussinesq approximation is invoked for the fluid effects under the variation of density with temperature and the resulting buoyancy term couples the flow field to the temperature field. The governing equations for the unsteady two-dimensional natural convection of mass, momentum and energy in the enclosure (cavity) can be written in terms of the following non-dimensional variables and numbers:

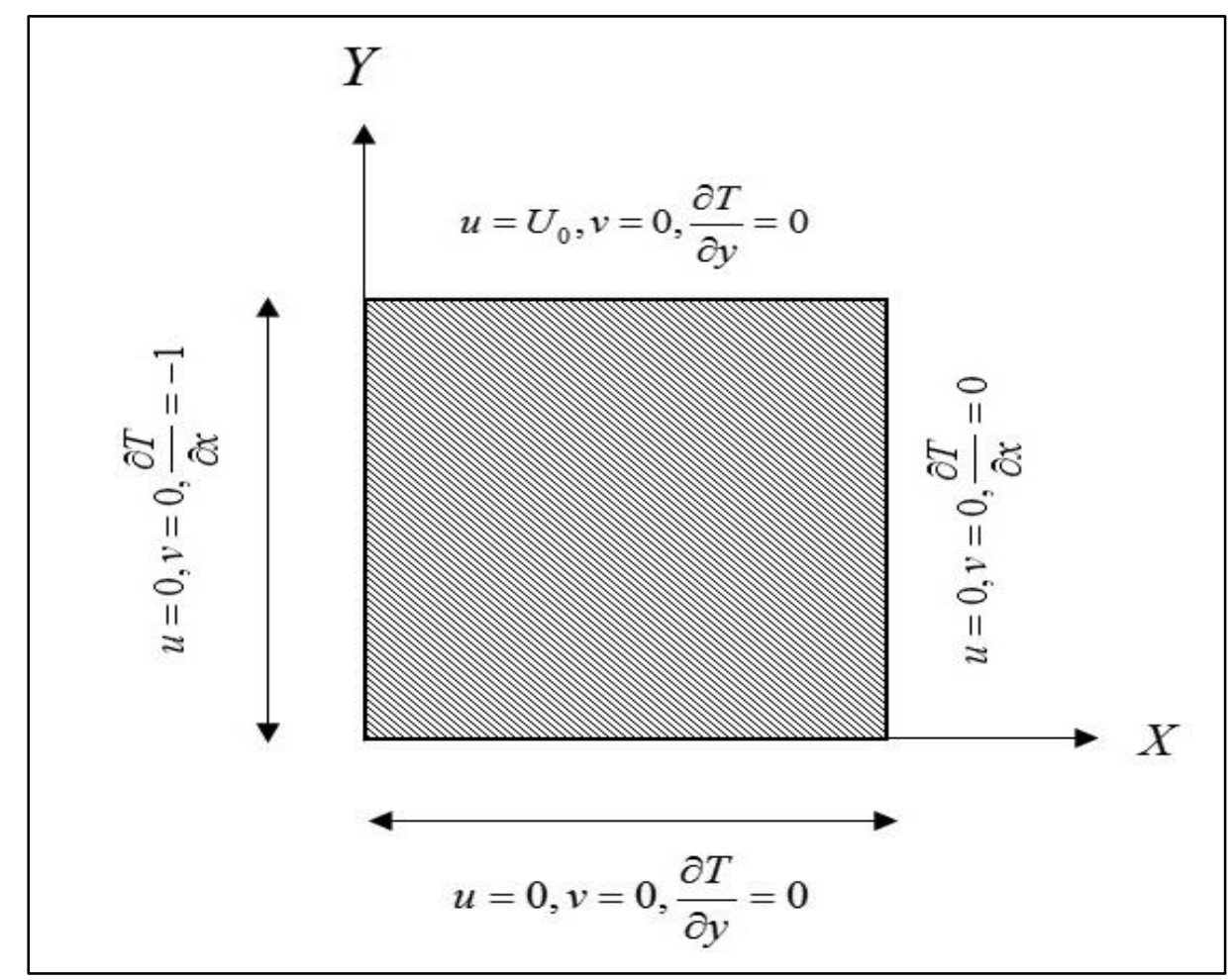

Fig.1. Schematic diagram of enclosure heat transfer system

$$
\frac{\partial u}{\partial x}+\frac{\partial v}{\partial y}=0
$$




$$
\begin{aligned}
& \frac{\partial u}{\partial t}+u \frac{\partial u}{\partial x}+v \frac{\partial u}{\partial y}=-\frac{\partial p}{\partial x}+\frac{1}{\operatorname{Re}}\left(\frac{\partial^{2} u}{\partial x^{2}}+\frac{\partial^{2} u}{\partial y^{2}}\right) \\
& \frac{\partial v}{\partial t}+u \frac{\partial v}{\partial x}+v \frac{\partial v}{\partial y}=-\frac{\partial p}{\partial y}+\frac{1}{G r^{2}} \frac{(T-1)}{T}+\frac{1}{\operatorname{Re}}\left(\frac{\partial^{2} v}{\partial x^{2}}+\frac{\partial^{2} v}{\partial y^{2}}\right) \\
& \frac{\partial T}{\partial t}+u \frac{\partial T}{\partial x}+v \frac{\partial T}{\partial y}=\frac{1}{\operatorname{Re} \operatorname{Pr}}\left(\frac{\partial^{2} T}{\partial x^{2}}+\frac{\partial^{2} T}{\partial y^{2}}\right)
\end{aligned}
$$

The transformed primary and secondary velocity and also thermal boundary conditions are:

$$
\begin{aligned}
& u(x, 1)=1, u(x, 0)=u=u(0, y)=u(1, y)=0, \\
& v(x, 1)=v(x, 0)=v(0, y)=v(1, y)=0, \\
& \frac{\partial T}{\partial x}(0, y)=-1, \frac{\partial T}{\partial x}(1, y)=0, \frac{\partial T}{\partial y}(x, 0)=\frac{\partial T}{\partial y}(x, 1)=0
\end{aligned}
$$

The dimensionless variables and parameters are defined as:

$$
\begin{aligned}
& x=X / H, y=Y / H, u=U / U_{o}, u=V / U_{o}, \theta=\frac{T-T_{c}}{T_{h}-T_{c}}, \\
& p=\frac{P}{\rho U_{0}^{2}}, \operatorname{Pr}=\frac{v}{\alpha}, \operatorname{Re}=\frac{U_{o} L}{v}, G r=\frac{g \beta\left(T_{h}-T_{c}\right) L^{3}}{v^{2}}
\end{aligned}
$$

Here $X$ is dimensionless $x$ - coordinate, $Y$ is dimensionless $y$-coordinate, $U$ is dimensionless $x$ direction velocity, $V$ is dimensionless $y$-direction velocity, $\theta$ is dimensionless temperature function, $P$ is dimensionless pressure, $P r$ is Prandtl number, $R e$ is Reynolds number, $G r$ is Grashof number, $T_{c}$ is cold wall temperature, $T_{h}$ is hot wall temperature, $T$ is dimensional temperature, $p$ is dimensional pressure, $g$ is gravity, $\rho$ is fluid density, $\alpha$ is thermal diffusivity, $v$ is kinematic viscosity, $H$ is height of enclosure (cavity wall dimension), $t$ is dimensional time. We note that the emerging thermal Grashof number encompasses the relative influence of gravity (buoyancy) forces to viscous forces in the regime. Further details are elaborated in Mohamad and Viskanta [27].

\section{MARKER-AND-CELL (MAC) NUMERICAL SOLUTION AND VALIDATION}

The momentum and energy balance equation i.e. Eqns. (2)-(4) have been solved using the MAC Method [26]. The continuity equation [Eqn. (1)] is used as a constraint due to mass conservation and this constraint may be used to obtain the pressure distribution. The numerical 
solution obtained in terms of the velocity components $(u, v)$ and stream functions $(\psi)$ are evaluated using the relationship between the stream function $(\psi)$ and the velocity components [28]. Here the stream function $(\psi)$ is defined in the usual way as per the Cauchy-Riemann equations, $u=\frac{\partial \psi}{\partial y}$ and $v=-\frac{\partial \psi}{\partial x}$. It may be noted that the positive sign of $\psi$ denotes anticlockwise circulation and therefore clockwise circulation is represented by the negative sign of $\psi$. In the MAC approach although we consider viscous flow, viscosity is not actually required for numerical stability [26]. Cell boundaries are labelled with half-integer values in the finite difference discretization. The marker particles do not participate in the calculation. Here we elaborate on the numerical discretization procedure. Based on the weak conservative form of the unsteady two-dimensional Navier-Stokes equations and heat conservation equation as defined by eqns. (1)-(4), we implement a grid meshing procedure using the following notation at the centre of a cell:

$$
u_{i-1 / 2, j}=\frac{1}{2}\left[u_{i-1, j}+u_{i, j}\right]
$$

Applying to the $\mathrm{x}$-direction momentum conservation eqn. (2) we have:

\section{Discretized Advection term:}

$$
\frac{\partial(u u)}{\partial x}=\frac{u u 1-u u 2}{\Delta x}
$$

where

$$
\begin{aligned}
& u u 1=\left[\frac{1}{2}\left(u_{i, j}+u_{i+1, j}\right)\right]^{2} \\
& u u 2=\left[\frac{1}{2}\left(u_{i-1, j}+u_{i, j}\right)\right]^{2}
\end{aligned}
$$

\section{Discretized Advection term:}

Similarly we have:

$$
\frac{\partial(u v)}{\partial y}=\frac{u v 1-u v 2}{\Delta y}
$$

where

$$
\begin{aligned}
& u v 1=\frac{1}{2}\left(u_{i, j}+u_{i, j+1}\right) \cdot \frac{1}{2}\left(v_{i, j}+v_{i+1, j}\right) \\
& u v 2=\frac{1}{2}\left(u_{i, j}+u_{i, j-1}\right) \cdot \frac{1}{2}\left(v_{i, j-1}+v_{i+1, j-1}\right)
\end{aligned}
$$


The following central difference formula are used for the second order derivatives:

$$
\begin{aligned}
& \nabla^{2} u=\frac{\partial^{2} u}{\partial x^{2}}+\frac{\partial^{2} u}{\partial y^{2}} \\
& \nabla^{2} u=\frac{u_{i-1, j}-2 u_{i, j}+u_{i+1, j}}{\Delta x^{2}}+\frac{u_{i, j-1}-2 u_{i, j}+u_{i, j+1}}{\Delta y^{2}}
\end{aligned}
$$

Applying to the y-direction momentum conservation eqn. (3) we have:

\section{Advection term:}

$$
\frac{\partial(v u)}{\partial x}=\frac{v u 1-v u 2}{\Delta x}
$$

Here the following notation applies:

$$
\begin{aligned}
& u v 1=\frac{1}{2}\left(u_{i, j+1}+u_{i, j}\right) \cdot \frac{1}{2}\left(v_{i, j}+v_{i+1, j}\right) \\
& u v 2=\frac{1}{2}\left(u_{i-1, j+1}+u_{i-1, j}\right) \cdot \frac{1}{2}\left(v_{i, j}+v_{i-1, j}\right) \\
& \frac{\partial(v v)}{\partial y}=\frac{v v 1-v v 2}{\Delta y} \\
& v v 1=\left[\frac{1}{2}\left(v_{i, j+1}+v_{i, j}\right)\right]^{2} \\
& v v 2=\left[\frac{1}{2}\left(v_{i, j-1}+v_{i, j}\right)\right]^{2}
\end{aligned}
$$

The central difference formula for the Laplacean operator is given by:

$$
\begin{aligned}
& \nabla^{2} v=\frac{\partial^{2} v}{\partial x^{2}}+\frac{\partial^{2} v}{\partial y^{2}} \\
& \nabla^{2} v=\frac{v_{i-1, j}-2 v_{i, j}+v_{i+1, j}}{\Delta x^{2}}+\frac{v_{i, j-1}-2 v_{i, j}+v_{i, j+1}}{\Delta y^{2}}
\end{aligned}
$$

Effectively the $x$-momentum equation discretization technique can be summarized as:

$$
u_{-} t p=u^{n}+d t *\left(-A+1 / \mathrm{Re}^{*} D 2 \_u\right)
$$

Where $A=$ advection term, $D 2 \_u=$ diffusion term $=$ Laplacian of $u$. There is a slight modification needed in the $y$-momentum equation due to the addition of a new term. Therefore this term must be included in the discretized equation and we have: 


$$
v-t p=v^{n}+d t^{*}\left(-B+1 / \operatorname{Re}^{*} D 2 \_v+1 / G r^{\wedge} 2 *(T-a) / T\right)
$$

Where $B=$ advection term, $D 2 \_v=$ diffusion term=Laplacian of $v$. It is further noteworthy that the temperature term $T$ is co-located such that it coincides with velocity before using it in the above equation to account for the staggered grid. After $u_{-} t p$ and $v-t p$ are projected to get $u$ and $v$, we can use the discretized temperature equation to get $T$ at next time level $\left(T^{n+1}\right)$ via the algorithm:

$$
T^{n+1}=T^{n}+d t^{*}\left(-A_{-} T+1 /\left(\operatorname{Re}^{*} \operatorname{Pr}\right) * D 2 \_T\right)
$$

where $A_{-} T=$ advection term, $D 22_{-} v=$ diffusion term=Laplacian of $T$. Next, we integrate in time by an incremental step $d t$ in each iteration until the final time $t=1.0$ is reached. The variables are co-located and plotted. Modern variants of the MAC method utilize the conjugate gradient schemes which solve the Poisson equation. Further details are provided in Basak et al. [29] and Amsden and Harlow [30]. To confirm mesh independence a grid-independence study is conducted. In computational fluid dynamics, of which finite difference simulation is merely one methodology, once a mesh provides a solution which is invariant with the finer meshes, the coarser mesh can be adopted. This reduces computational cost but retains the necessary accuracy. Table 1 shows that accuracy to three decimal places is achieved for Nusselt number at the left wall with a mesh of $61 \times 61$ which is sufficient for heat transfer computations and therefore this is adopted for all subsequent simulations.

\begin{tabular}{|c|c|}
\hline Grid size & Average Nusselt number $(\mathrm{Nu})$ \\
\hline $21 \times 21$ & 0.141527 \\
\hline $41 \times 41$ & 0.145274 \\
\hline $51 \times 51$ & 0.145483 \\
\hline $61 \times 61$ & 0.146316 \\
\hline $71 \times 71$ & 0.146956 \\
\hline
\end{tabular}

\section{Table 1: Grid independent study}

Furthermore to corroborate the present computations, visualizations of the temperature (isotherm) and streamline distributions for two special cases have been provided. These replicate the solutions of Basak et al. [29]. The equivalent Rayleigh number used in [29] is merely the product of the thermal Grashof number and Prandtl number used in the present model (i.e. $R a=G r P r$ ). Generally very close correlation is attained, as observed in Figs. 2 and 3 and confidence in the present MAC computational code is therefore justifiably high. 


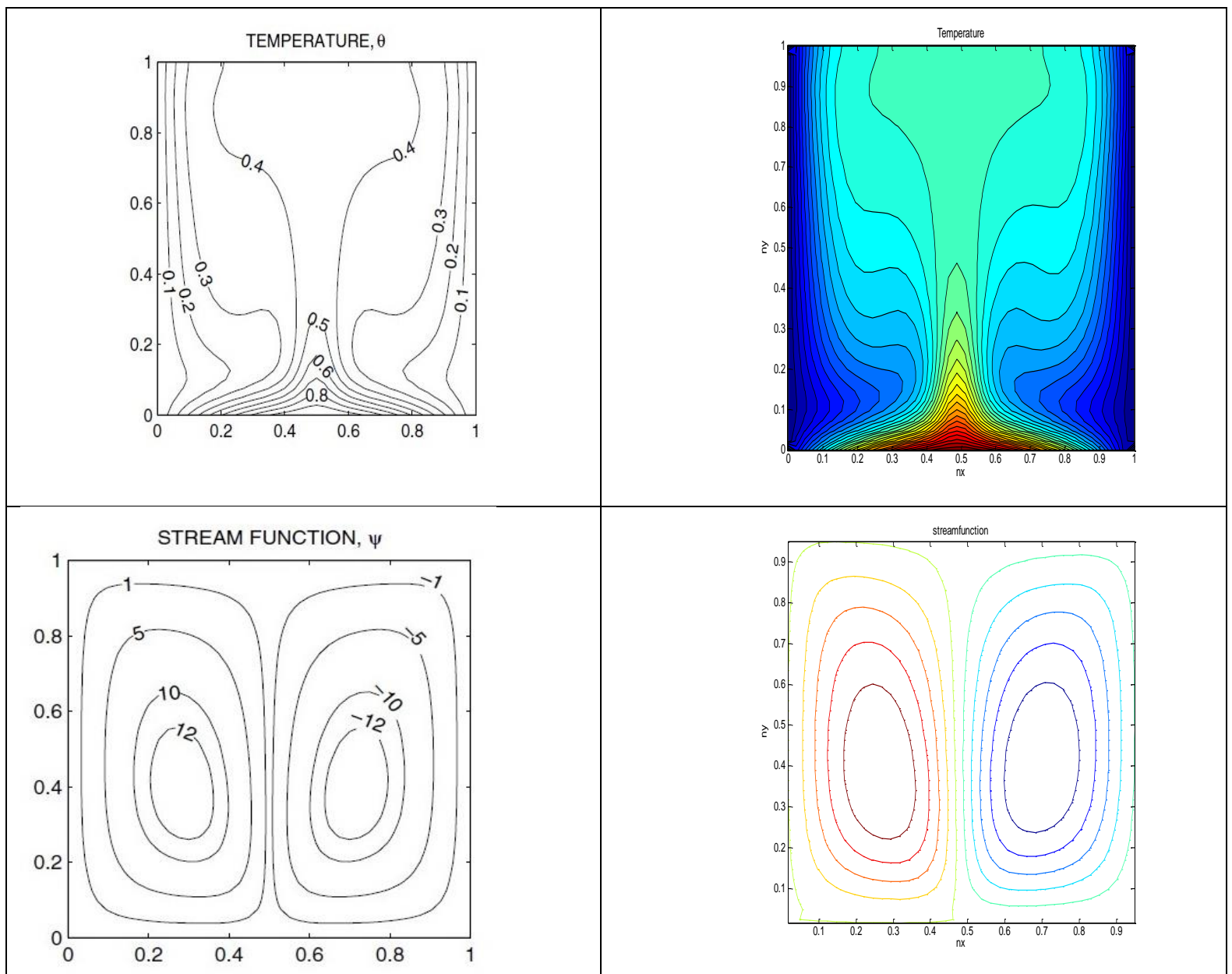

Fig 2: Comparison contour plots, non-uniform bottom heating, $T=\sin (\pi x), \operatorname{Pr}=10, \operatorname{Ra}=10^{5}$.

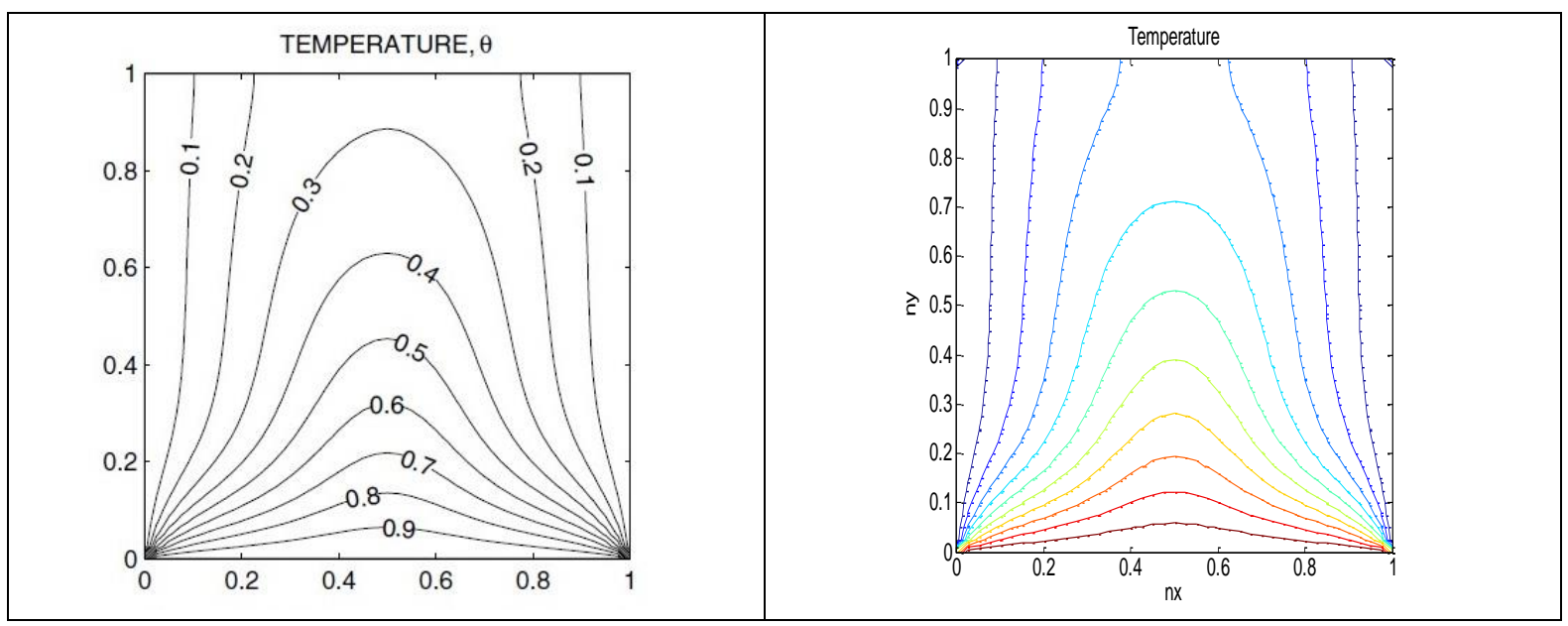

Fig. 3: Contour plots, non-uniform bottom heating, $T=1$, with $\operatorname{Pr}=0.7$ and $R a=5 \times 10^{5}$.

\section{MAC NUMERICAL RESULTS AND DISCUSSION}

Extensive numerical computations have been performed and are illustrated in Figs. 4a-d to 11a-d. In each plot we visualize the stream function, temperature, vorticity and pressure. The 
left wall of square cavity is maintained at a constant heat flux the remaining walls are thermally isolated. In figs. 4a-d to 6a-d thermal Grashof number $(G r)$ is varied keeping $\operatorname{Pr}=0.7$ (air) and $R e=10$ (laminar). The results are depicted as stream lines, isotherms (temperatures), vorticity and pressure profiles.
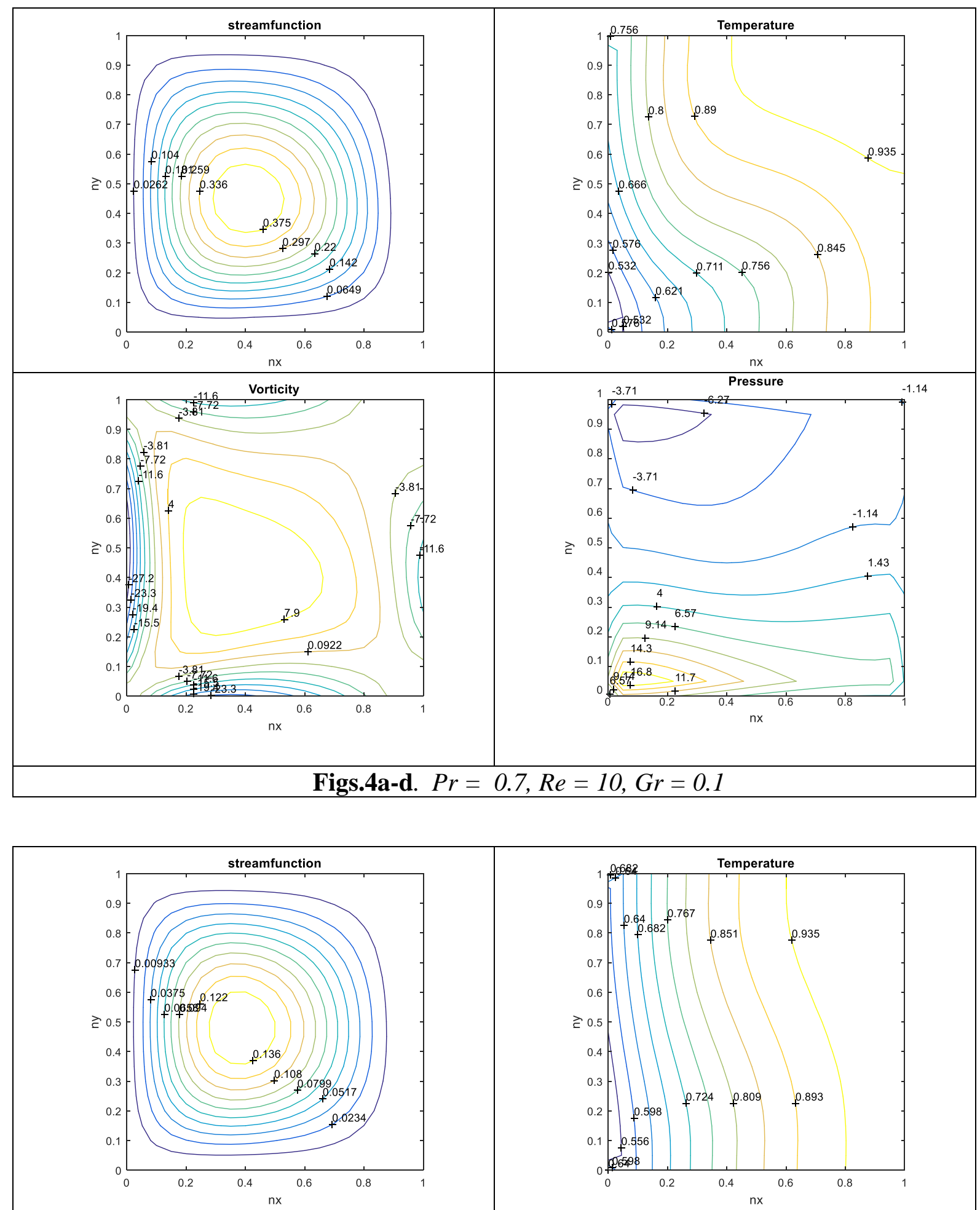


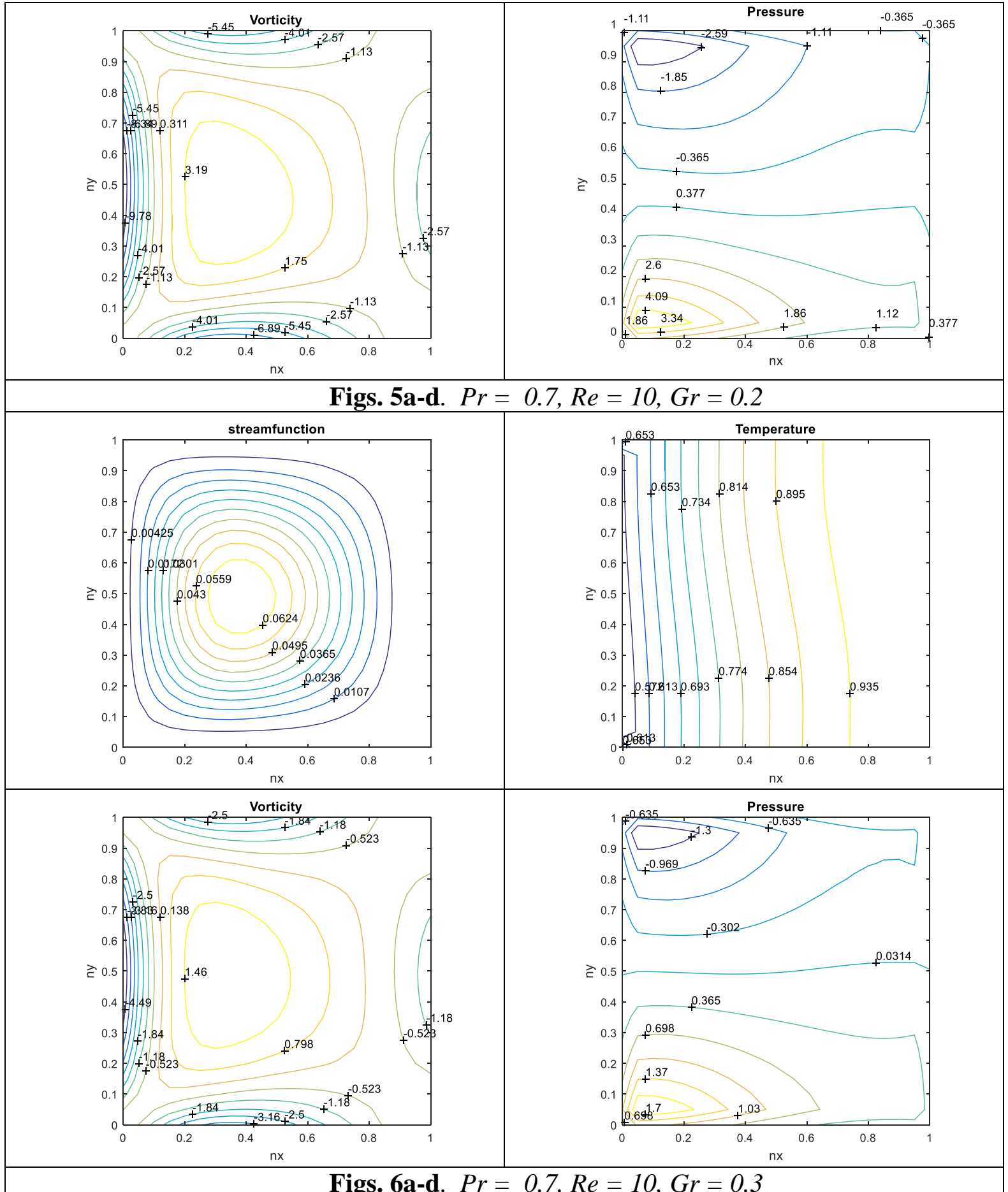

Figs. 6a-d. $\operatorname{Pr}=0.7, \operatorname{Re}=10, \mathrm{Gr}=0.3$ 


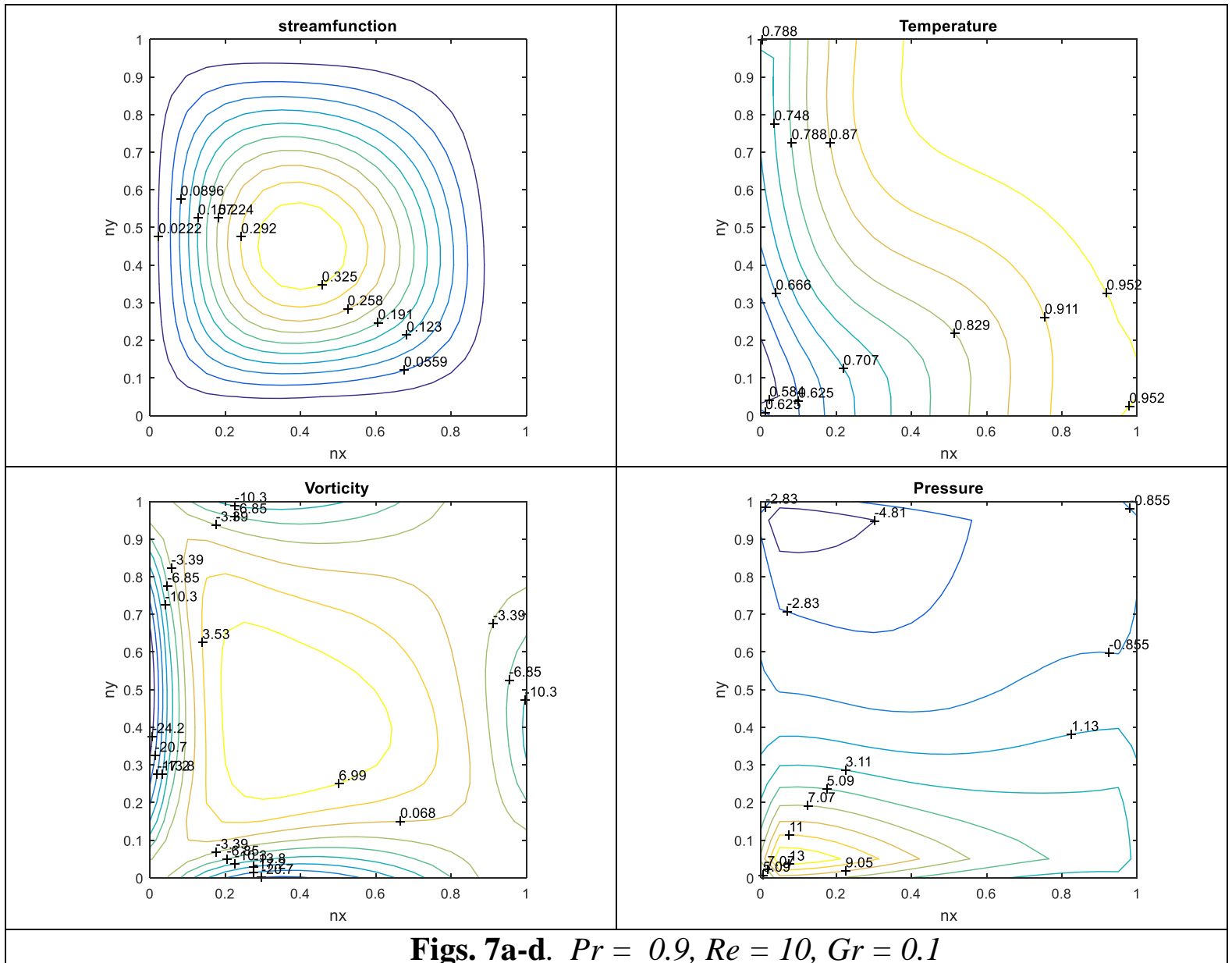

Figs. 7a-d. $\operatorname{Pr}=0.9, \operatorname{Re}=10, G r=0.1$

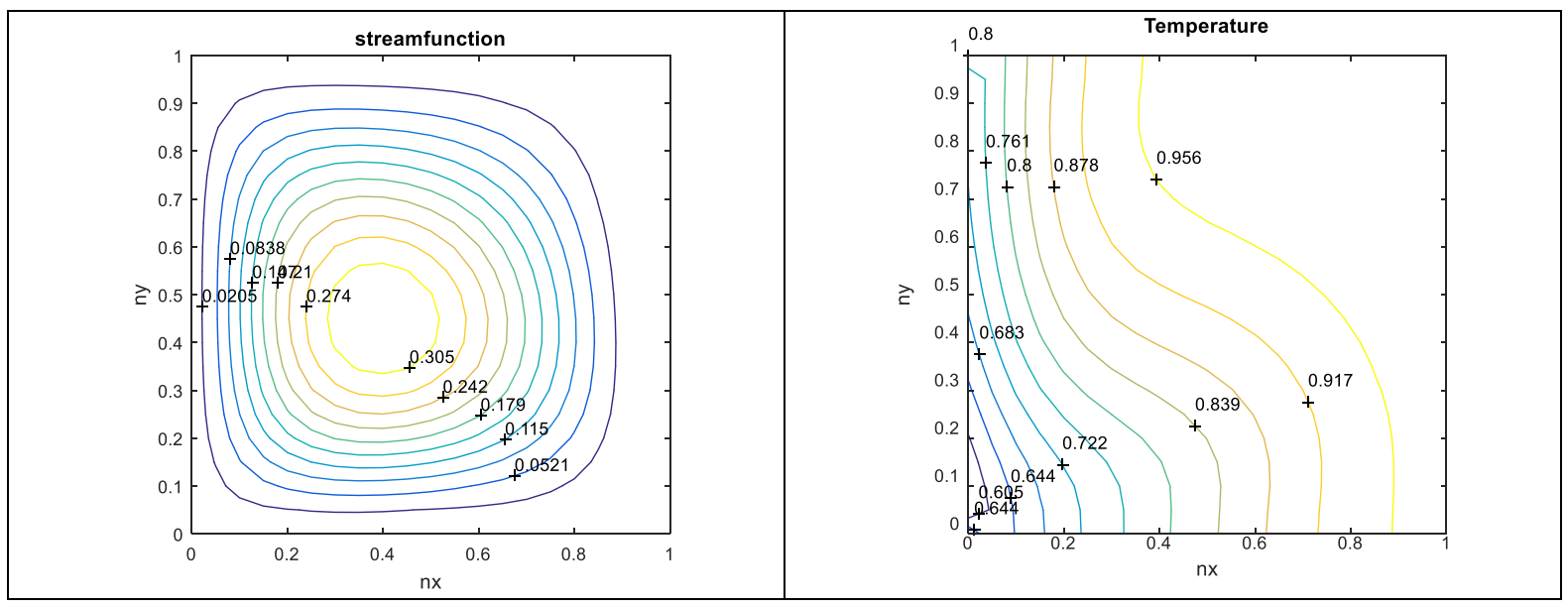



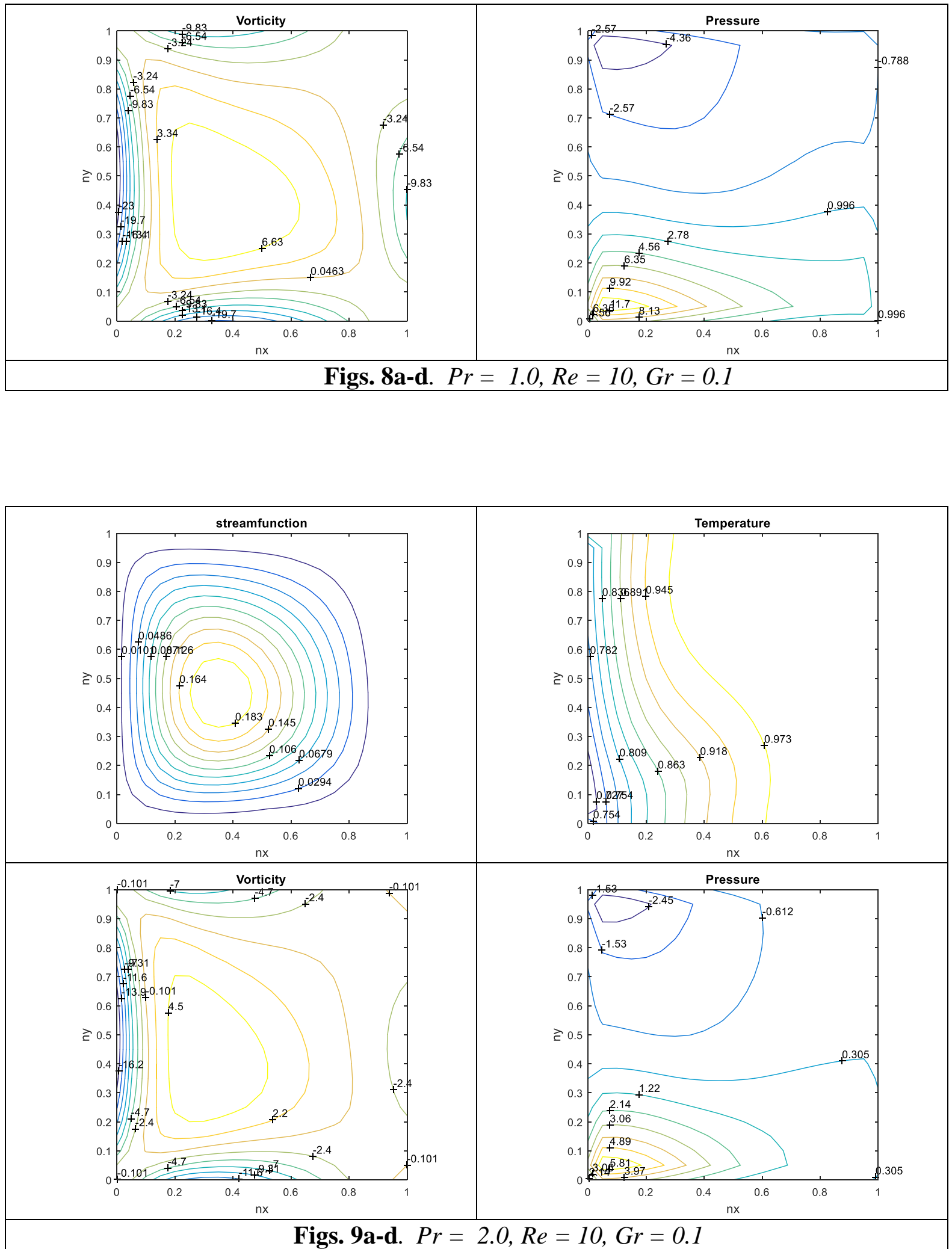


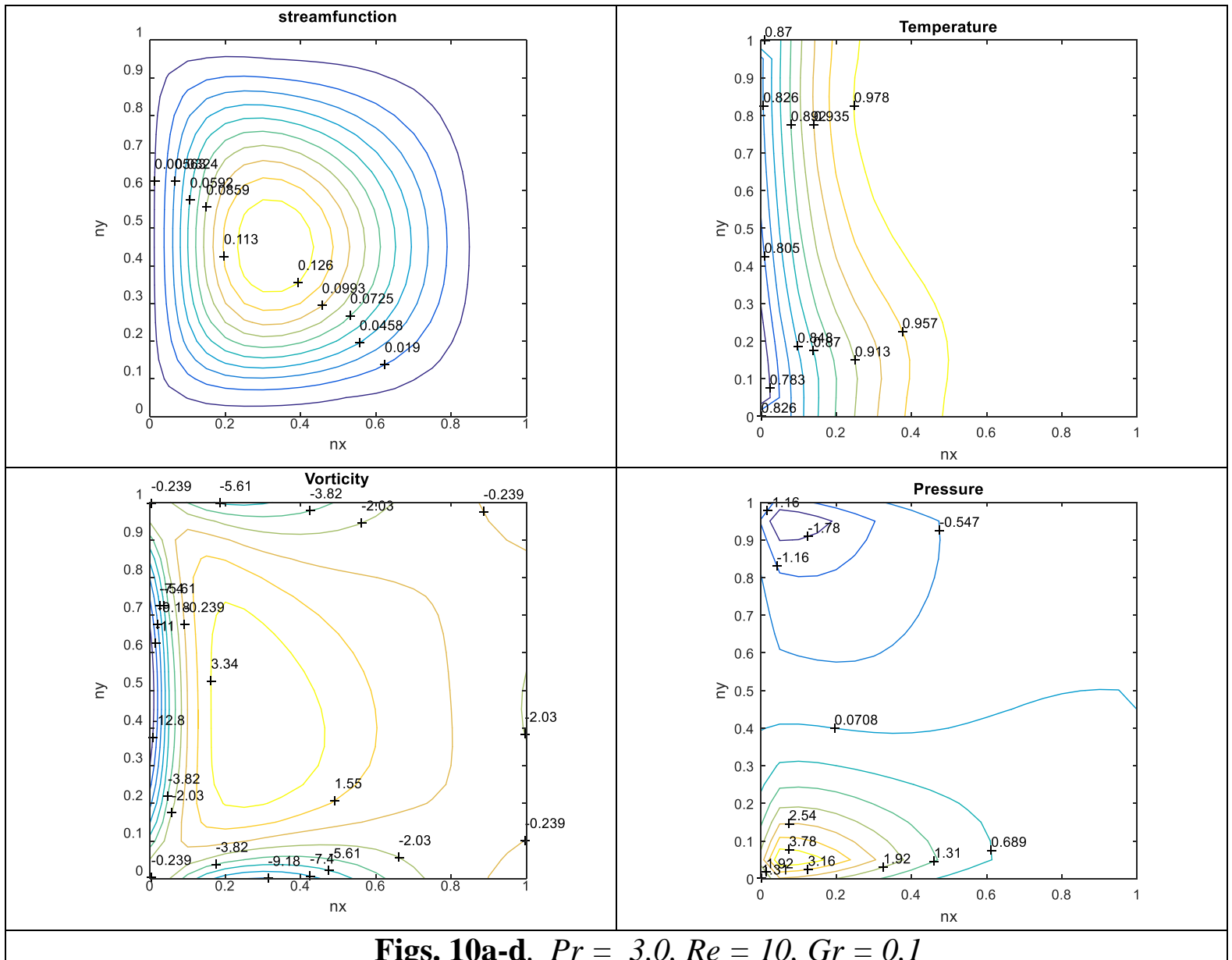

Figs. 10a-d. $\operatorname{Pr}=3.0, \operatorname{Re}=10, G r=0.1$

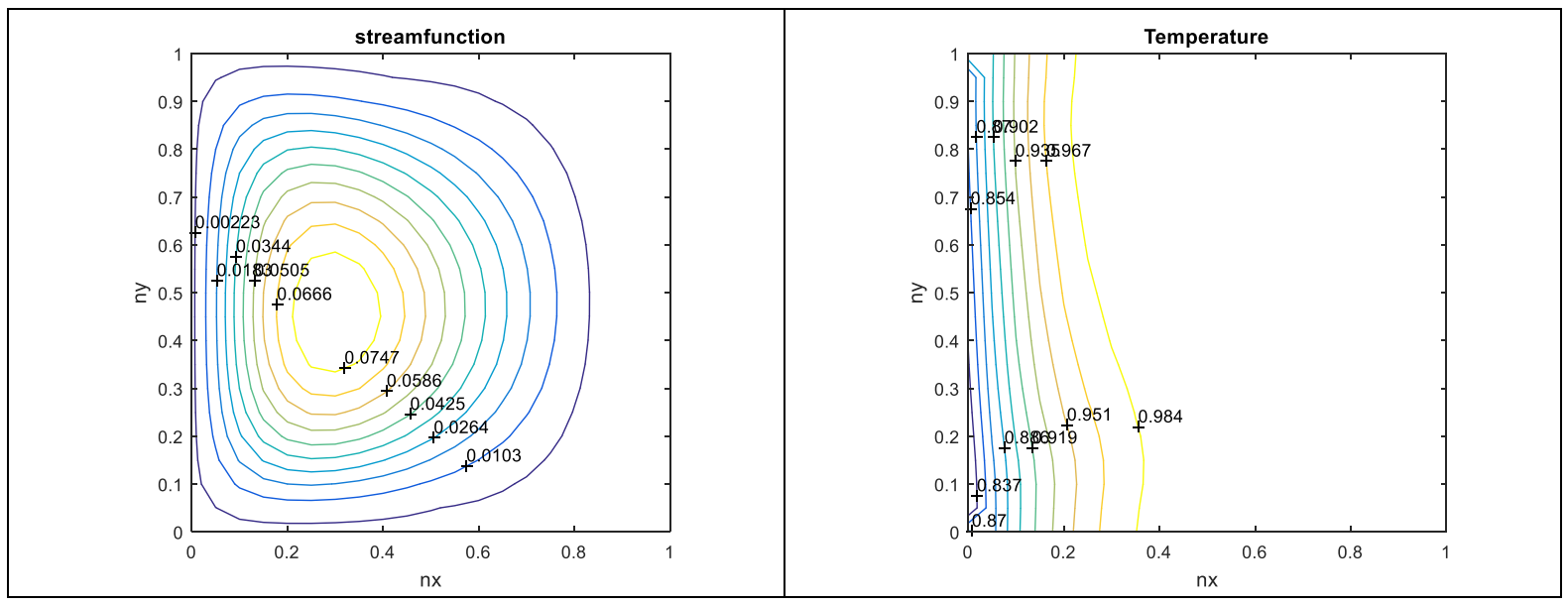




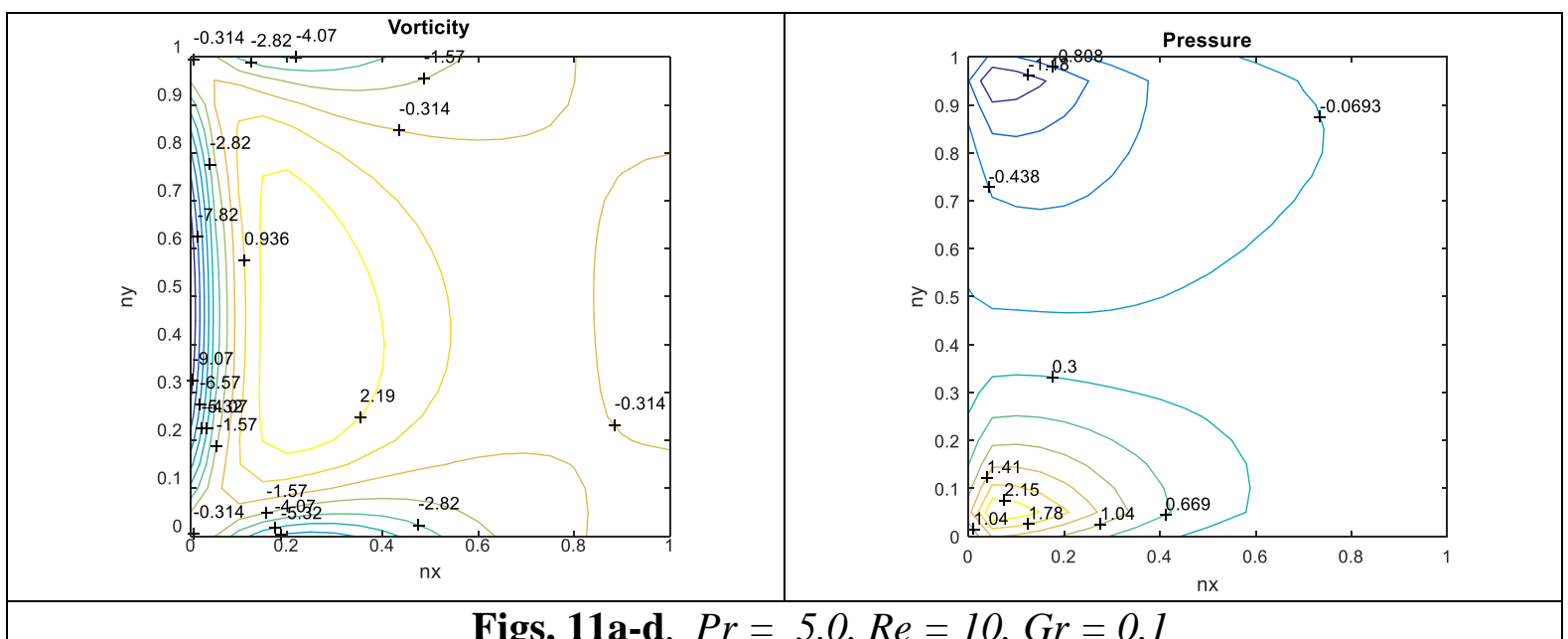

Figs. 11a-d. $\operatorname{Pr}=5.0, \operatorname{Re}=10, \mathrm{Gr}=0.1$

To establish the influence of thermal Grashof number on each of the four distributions, respective comparisons have to be made as follows: For stream function which defines velocity distribution in the cavity we compare Fig $4 \mathrm{a}(\mathrm{Gr}=0.1)$ versus Fig $5 \mathrm{a}(\mathrm{Gr}=0.2)$ and Fig $6 \mathrm{a}$ $(G r=0.3)$. For temperature (isotherms) which define thermal convection pattern in the enclosure we compare Fig $4 \mathrm{~b}(G r=0.1)$ versus Fig $5 \mathrm{~b}(G r=0.2)$, Fig $6 \mathrm{~b}(G r=0.3)$. For vorticity which defines intensity of circulation in the cavity we compare Fig 4c $(G r=0.1)$ versus Fig 5c $(G r=0.2)$ and Fig 6c $(G r=0.3)$. For pressure evolution in the enclosure we compare Fig 4d $(G r=0.1)$ versus Fig $5 d(G r=0.2)$ and Fig 6d $(G r=0.3)$. With increasing Grashof number there is a decrease in the streamline contour magnitudes. Primary circulation generally occupies most of the square cavity, although it is slightly biased towards the lower left corner. This is probably attributable to the constant heat flux condition imposed at the left wall. The strength of the circulation is weakened with greater thermal Grashof number. For example in the interior core of the streamline patterns, the maximum value of stream function is progressively decreased from 0.375 to 0.136 and thereafter to 0.0624 . The thermal Grashof number features in the thermal buoyancy coupling term, $\frac{1}{G r^{2}} \frac{(T-1)}{T}$, in the dimensionless momentum conservation equations. Increasing values of this parameter correspond to an enhancement in thermal buoyancy effect relative to viscous hydrodynamic effect. Since $G r<1$ in all computations, there is a dominance of viscous force in the enclosure over thermal buoyancy force. This serves to damp the flow and to decrease velocities, manifesting in depletion in streamline contour values. The general structure of streamlines is not hugely modified although there is widening in the gaps between contours which is characteristic of decelerating flow in the cavity. With an increment in thermal Grashof number the temperature 
magnitudes (isotherms) are intensified towards the left wall. There is a strong distortion at $\mathrm{Gr}$ $=0.1$ (fig. $4 \mathrm{~b}$ ) which is homogenized in fig. $5 \mathrm{~b}$ and even more so in fig. $5 \mathrm{~d}$ for $G r=0.3$. The effective tripling in the thermal buoyancy force therefore stabilizes temperature circulation and orientates the temperature distribution towards the left wall. Isotherms become increasingly parallel to the left wall and the deflected contours observed at low thermal Grashof number are all but eliminated. However isotherm magnitudes are reduced and this is also associated frequently with stronger thermal buoyancy effect, as described by Gebhart et al. [31]. The isotherm magnitude reduces from 0.756 (fig. 4b) to 0.682 (fig. 5b) and plummets to the minimum of 0.653 (fig. 6b). Effectively thermal buoyancy can be utilized to cool the enclosure regime and this has important implications in electronic systems. However in solar collectors where the opposite effect is desired weaker buoyancy is critical to achieving higher temperatures in the enclosure (collector). There is a substantial reduction in vorticity magnitudes also with increasing Grashof number. The retardation induced with greater thermal buoyancy also reduces the intensity of circulation in the enclosure which decreases iso-vorticity contour magnitudes. We observe that the maximum value associated with the inner core of the flow falls from 7.9 (fig. 4c) to 3.19 (fig. 5c) and finally the lowest value of 1.46 (fig 6c). Again it is evident that there is skewness in the contours towards the left wall. The contour gaps increase and there is a shrinking in the inner circulation zone with greater thermal Grashof number. Pressure magnitudes are however markedly increased with greater thermal Grashof number in the upper left zone of the enclosure whereas they are depressed in the lower left zone and everywhere else. In the top left corner of the cavity, for $G r=0.1$ (fig. $4 \mathrm{~d}$ ) the pressure is 3.71 , for $G r=0.2$ (fig. $5 \mathrm{~d}$ ) it is -1.11 and for $G r=0.3$ (fig. $6 \mathrm{~d}$ ) the values becomes positive i.e. +0.635 , indicating an ascent in pressures. Conversely at the bottom left corner pressures fall from a maximum of 16.8 , to 4.09 and eventually to 1.7 , constituting a significant pressure reduction in this location. The influence of thermal Grashof number is therefore impactful on all characteristics of the enclosure flow.

In figs. 7a-d to 11a-d Prandtl number $(\mathrm{Pr})$ is varied keeping $R e=10$ (laminar) and $G r=0.1$ (i.e. very weak thermal buoyancy). Again to acquire a proper perspective of the influence of $P r$ which is a characteristic property of the fluid, inspection of the graphs is conducted as follows. For stream function which defines velocity distribution in the cavity we compare Fig $7 \mathrm{a}(\mathrm{Pr}=$ $0.9)$ versus Fig 8a $(\operatorname{Pr}=1.0)$, Fig 9a $(\operatorname{Pr}=2.0)$, Fig 10a $(\operatorname{Pr}=3.0)$ and Fig 11a $(\operatorname{Pr}=5.0)$. For temperature (isotherms) which defines convective heat transfer convection pattern in the enclosure we compare Fig $7 \mathrm{~b}(\operatorname{Pr}=0.9)$ versus Fig 8b $(\operatorname{Pr}=1.0)$, Fig $9 \mathrm{~b}(\operatorname{Pr}=2.0)$, Fig 10b $(P r=3.0)$ and Fig $11 \mathrm{~b}(\operatorname{Pr}=5.0)$. For vorticity which quantifies the intensity of circulation 
patterns in the enclosure we compare Fig $7 \mathrm{c}(\operatorname{Pr}=0.9)$ versus Fig $8 \mathrm{c}(\operatorname{Pr}=1.0)$, Fig $9 \mathrm{c}(\operatorname{Pr}=$ 2.0), Fig 10c $(\operatorname{Pr}=3.0)$ and Fig 11c $(\operatorname{Pr}=5.0)$. For pressure distribution in the enclosure we compare Fig 7d $(\operatorname{Pr}=0.9)$ versus Fig 8d $(\operatorname{Pr}=1.0)$, Fig 9d $(\operatorname{Pr}=2.0)$, Fig 10d $(\operatorname{Pr}=3.0)$ and Fig 11d $(\operatorname{Pr}=5.0)$. The Prandtl number is the ratio of momentum diffusivity to the thermal diffusivity. Larger values of Prandtl number correspond to the case of less heat transfer from the boundary to the fluid. Prandtl number is also the product of dynamic viscosity and specific heat capacity divided by the thermal conductivity of the fluid. With Prandtl number lower than unity, the momentum diffusion rate is exceeded by the thermal (energy) diffusion rate. When $\operatorname{Pr}$ attains unity value both heat and momentum are diffused at equal rates (momentum and thermal boundary layer thicknesses at the enclosure walls are equivalent). When $\operatorname{Pr}$ exceeds unity, the momentum diffusion rate exceeds thermal diffusion rate. Inspection of figs 5a-9a indicates that stream function magnitudes are progressively reduced with an increase in Prandtl number. For example in the inner core of the circulating fluid, where maximum stream function values arise on the streamline plots, magnitudes decrease from $+0.325(\operatorname{Pr}=0.9)$ through $+0.305(\operatorname{Pr}=1.0),+0.183(\operatorname{Pr}=2.0),+0.128(\operatorname{Pr}=3.0)$ to finally $+0.0747(\operatorname{Pr}=5.0)$. The separation of the streamline contours simultaneously increases indicating a deceleration in the circulating flow. With greater Prandtl number the heat transfer from the left wall to the body of fluid and therefore to the right wall is enhanced. The higher Prandtl number corresponds to greater thermal conductivity of the fluid which encourages thermal diffusion and boosts temperatures as observed in Figs $7 \mathrm{~b}, 8 \mathrm{~b}, 9 \mathrm{~b}, 10 \mathrm{~b}$ and $11 \mathrm{~b}$. Isotherms are systematically drawn closer to the left wall and also to each other indicating that the flow is energized with greater Prandtl number. Heat transfer rates to the walls are therefore reduced. With an increase in Prandtl number, the vorticity is also stifled significantly. This is both in the core flow and also at other locations. The maximum vorticity is located in the core flow (which is skewed towards the left wall of the cavity). The core flow region shrinks with increasing Prandtl number. Magnitudes here drop from +6.99 (positive values imply anti-clockwise circulation) for $\mathrm{Pr}=$ 0.9 through $+6.63(\operatorname{Pr}=1.0)$, to $+4.5(\operatorname{Pr}=2.0)$ to $+3.34(\operatorname{Pr}=3.0)$ to eventually $+2.19(\operatorname{Pr}=$ 5.0). There is concurrently a growth in vortex pattern in the right wall proximity as the vorticity is decreased in the left zone of the enclosure. However the strength of the newly developing zones is decreased i.e. vorticity magnitudes still are reduced with increase in Prandtl number even in the right wall zone of the enclosure. Finally a reduction in pressure contour magnitudes is also observed with increasing Prandtl number (figs. 7d-11d). Pressure contours continuously diverge from each other as this occurs. 


\section{CONCLUSIONS}

A numerical investigation of unsteady heat and momentum transfer in a Newtonian fluid occupying a square cavity has been conducted. The non-dimensional conservation equations have been solved with the Harlow-Welch Marked and Cell (MAC) finite difference approach. The square cavity has been simulated with a constant heat flux at the left wall and thermal insulation at all other walls. A grid independence study has been conducted. Also solutions have been validated with earlier published computations for special base wall heating conditions. The influence of several control parameters has been elucidated via streamline, temperature (isotherm), vorticity and pressure plots. The computations have shown that:

(i) With an increment in thermal Grashof number (i.e. buoyancy effect) there is a reduction in streamline contour magnitudes (i.e. flow deceleration), an increase in temperature magnitudes (isotherms) and an associated decrease in wall heat transfer rates, a suppression of vorticity and intensity of circulation and an enhancement in pressure magnitudes in the upper left zone of the enclosure.

(ii) With increasing Prandtl number, there is a decrease in stream function magnitudes (flow retardation), increase in isotherm magnitudes (i.e. heating of the enclosure fluid) and associated depression in wall heat transfer rates, a reduction in vorticity (and therefore inhibition of circulation in the cavity) and finally a suppression in pressure contour magnitudes.

The MAC numerical scheme apparently achieves efficient and accurate solutions for transient enclosure thermal convection. The current study has however been confined to Newtonian fluids and has ignored thermal radiation. Non-Newtonian working fluids e.g. micropolar [5] and radiative flux models [2] are also of interest in optimizing solar collector thermal performance [32] and will be addressed in the near future.

\section{REFFERENCES}

[1] Y. Hasemi, Tokunaga, T., Some experimental aspects of turbulent diffusion flames and buoyant plumes from fire sources against a wall and in a corner of walls, Combustion Science and Technology, 40 (1984) 1-18.

[2] O. Anwar Bég, N. Ali, A. Zaman, Eemaan T. A. Bég and Ayesha Sohail, Computational modelling of heat transfer in annular porous medium solar energy absorber with a P1-radiative differential approximation, J. Taiwan Inst. Chemical Eng. (2016). doi.org/10.1016 j.jtice.2016.06.034 (11 pages). 
[3] E. M. Llanos, S. J. Zarrouk, R. A. Hogarth, Numerical model of the Habanero geothermal reservoir, Australia, Geothermics, 53 (2015) 308-319.

[4] A. Y. Gelfgat, On three-dimensional instability of a traveling magnetic field driven flow in a cylindrical container, J. Crystal Growth, 279 (2005) 276-288.

[5] R. Bhargava, S. Sharma, P. Bhargava, O. Anwar Bég and A. Kadir, Finite element simulation of nonlinear convective heat and mass transfer in a micropolar fluid-filled enclosure with Rayleigh number effects, Int. J. Applied Computational Mathematics (2016). DOI: 10.1007/s40819-016-0180-9 (33pages.

[6] N. Rudraiah, R.M. Barron, M. Venkatachalappa, C.K. Subbaraya, Effect of magnetic field on free convection in a rectangular enclosure, Int. J. Eng.Sci., 33 (1995) 1075-1084.

[7] J.P, Garander, T. Alboussiere, R. Moreau, Buoyancy driven convection in a rectangular enclosure with a transverse magnetic field, Int. J. Heat Mass Transfer 35 (1992) 741- 748.

[8] M.S. Bhavaneswari, S. Sivasankaran and Y. J. Kim, Numerical study on double diffusive mixed convection with a soret effect in a two-sided lid-driven cavity, Numer. Heat. Trans. A., 59 (2011) 543-560.

[9] M. K. Moallemi, Jang K.S. Prandtl number effects on laminar mixed convection heat transfer in a lid-driven cavity, Int J Heat Mass Transfer, 35 (1992) 1881-92.

[10] M. Sathiyamoorthy, Ali J.Chamkha, Natural convection flow under magnetic field in a square cavity for uniformly (or) linearly heated adjacent walls, Int. J. Numerical Methods for Heat \& Fluid Flows, 22 (2012) 677-698.

[11] S.C Kakarantzas, Sarris, I.E., Grecos, A.P and Vlachos, N.S, Magnetohydrodynamic natural convection in a vertical cylindrical cavity with sinusoidal upper wall temperature, Int. J. Heat and Mass Transfer, 28 (2009) 250-259.

[12] S. Saravanan, A.K. Abdul Hakeema, P. Kandaswamy, J. Leeb, Buoyancy convection in a cavity with mutually orthogonal heated plates, Int. J. Computers and Mathematics with Applications, 55 (2008) 2903-2912.

[13] K.L. Walker, G.M. Homsy, Convection in porous cavity, J. Fluid Mech., 87 (1978) 449474.

[14] A.K. Abdul Hakeema, S. Saravanan, P. Kandaswamy, Natural convection in a square cavity due to thermally active plates for different boundary conditions, Int. J. Computers and Mathematics with Applications, 62 (2011) 491-496.

[15] X. Shi, J.M. Khodadadi, Laminar natural convection heat transfer in a differentially heated square cavity due to a thin fin on the hot wall, ASME J. Heat Transf., 125 (2003) 624-634.

[16] E.K. Lakhal, M. Hasnaoui, E. Bilgen, P. Vasseur, Natural convection in inclined rectangular enclosures with perfectly conducting fins attached on the heated wall, Heat Mass Transf., 32 (1997) 365-373. 
[17] J.M. House, C. Beckermann, T.F. Smith, Effect of centered conducting body on natural convection heat transfer in an enclosure, Numer. Heat Transf. Part A, 18 (1990) 213-225.

[18] S. Saravanan, P. Kandaswamy, Free convection in an inclined enclosure with internal heat generation, in: Proc. 4th Asian Comp. Fluid Dyn. Conf., Mianyang, China (2000). 664669.

[19] M. Keyhani, L. Chen, D.R. Pitts, The aspect ratio effect on natural convection in an enclosure with protruding heat source, ASME J. Heat Transfer, 113 (1991) 883-891.

[20] T. Basak, S. Roy, A.J. Chamkha, A Peclét number based analysis of mixed convection for lid-driven porous square cavities with various heating of bottom wall, Int. Comm. Heat Mass Transfer, 39 (2012) 657-664.

[21] T. Basak, Abhishek Kumar Singh, T.P. Akshaya Sruthi, S. Roy, Finite element simulations on heat flow visualization and entropy generation during natural convection in inclined square cavities, Int. Comm. Heat and Mass Transfer,51 (2014) 1-8.

[22] Muneer A. Ismael, Ioan Pop b, Ali J. Chamkha, Mixed convection in a lid-driven square cavity with partial slip, Int. J. Thermal Sciences, 82 (2014) 47-61.

[23] M. Bhattacharya, T. Basak, H.F. Oztop, Y. Varol, Mixed convection and role of multiple solutions in lid-driven trapezoidal enclosures, Int. J. Heat. Mass Transf. 63 (2013) 366- 388.

[24] Saravanan S, Hakeem AKA, Kandaswamy P, Lee J. Buoyancy convection in a cavity with mutually orthogonal heated plates. Comput. Math. Appl., 55 (2008) 2903-12.

[25] Kandaswamy P, Lee J, Abdul Hakeem AK, Saravanan S. Effect of baffle-cavity ratios on buoyancy convection in a cavity with mutually orthogonal heated baffles, Int. J. Heat Mass Transfer, 51 (2008) 1830-7.

[26] F.H. Harlow and J.E. Welch, Numerical calculation of time-dependent viscous incompressible flow of fluid with free surface, Physics of Fluids, 8 (1965) 2182-2190.

[27] A. A. Mohamad and R. Viskanta, Flow and heat transfer in a lid-driven cavity filled with a stably stratified fluid, Appl. Math. Modelling, 19 (1995) 465-472.

[28] G.K.Batchelor, An Introduction to Fluid Dynamics, Cambridge University Press, UK (1967).

[29] T. Basak, S.Roy, A.R.Balakrishnan, Effects of thermal boundary conditions on natural convection flows within a square cavity, Int. J. Heat \& Mass Transfer, 49 (2006) 4525-4535.

[30] A.A. Amsden and F.H. Harlow, A simplified MAC technique for incompressible fluid flow calculations, J. Comp. Physics, 6 (1970) 322-325.

[31] B. Gebhart et al., Buoyancy-induced Flows and Transport, Hemisphere, Washington, USA (1988). 
[32] N. Lior, Thermal theory and modeling of solar collectors, Solar Collectors, Energy Storage, and Materials, F. de Winter, Ed., MIT Press, Cambridge, Massachusetts, USA (1990). 